\title{
QUANTIFICATION OF RETINAL NERVE FIBER LAYER FOR EARLY DETECTION OF ANTERIOR VISUAL PATHWAY LESIONS
}

\author{
Noureen Malik, Hannan Masud, Muhammad Manzoor Awan \\ Armed Forces Institute of Ophthalmology/National University of Medical Sciences (NUMS) Rawalpindi Pakistan
}

\begin{abstract}
Objective: To assess the role of quantification of retinal nerve fiber layer for early detection of anterior visual pathway lesions.

Study Design: Case-control study.

Place and Duration of Study: Armed Forces Institute of Ophthalmology, Rawalpindi, from Jan to Jul 2019.

Methodology: A total of 100 cases of anterior visual pathway lesions of varying clinical presentations were included in the study. There were 100 matched controls who were selected from the community without any ophthalmological abnormality using non-probability consecutive sampling technique. Retinal nerve fiber layer (RNFL) thickness was measured with the help of optical coherence tomography in both the cases and controls. Mean retinal nerve fiber layer values were compared in both the groups. Student's t-test was applied to look for any significant difference between the two groups.

Results: Mean age of the patients was $39.14 \pm 3.925$ years while mean age of the controls was $39.23 \pm 2.415$ years. Mean retinal nerve fiber layer thickness in the case group was $72.21 \pm 9.615 \mu \mathrm{m}$ while on the control group was $101.34 \pm 9.615 \mu \mathrm{m}$. A statistically significant difference was observed between cases and controls in terms of mean retinal nerve fiber layer thickness $(p<0.001)$. Subjects with retinal nerve fiber layer thickness $<85 \mu$ m were more likely to exhibit anterior visual pathway lesions (OR=15.915 [6.278-40.346]; 95\% CI, $p<0.001$ ).

Conclusion: Decreased retinal nerve fiber layer thickness can serve as a predictor for anterior visual pathway lesions. Optical coherence tomography should be incorporated for routine screening of high-risk cases in order to identify anterior visual pathway lesions in time.
\end{abstract}

Keywords: Anterior visual pathway, Optical coherence tomography, Retinal neurons.

This is an Open Access article distributed under the terms of the Creative Commons Attribution License (http://creativecommons.org/licenses/by/4.0), which permits unrestricted use, distribution, and reproduction in any medium, provided the original work is properly cited.

\section{INTRODUCTION}

Health system of Pakistan is in the developing phase and liaison between different specialties is still lacking. Ophthalmology needs collaboration with a lot of specialities of medicine as well as technical fields to grow and work better for the patients ${ }^{1}$. Neurology is one of those specialties which has a very close link with ophthalmology ${ }^{2}$. Retina has been considered as an extension of brain or neural tissue in the domain of eye ${ }^{3}$. Therefore, retinal parameters may be applied in detection of various neuro-ophthalmological diseases 4,5 . Optical coherence tomography (OCT) has revolutionized ophthalmology by giving the ophthalmologist the opportunity to analyze var-

Correspondence: Dr Noureen Malik, Dept of Ophthalmology, Armed Forces Institute of Ophthalmology, Rawalpindi Pakistan Received: 13 Jul 2019; revised received: 16 Aug 2019; accepted: 19 Aug 2019 ious structures and sub-structures of retina ${ }^{6}$. It is also useful in providing a non-invasive tool to diagnose a lot of neurological illnesses ${ }^{7}$. Patients with optic neuropathies also get benefit from this investigation technique both in terms of diagnosis and observing the response of treatment ${ }^{8}$. This multipurpose diagnostic modality has been utilized both in clinical settings and research purpose $^{9}$. OCT produces two-dimensional cross-sections of the retina with very good spatial resolution. The operation of the OCT is based on an optical measurement technique called "low coherence interference". When light emitted by the source of the device is directed to the eye, it is reflected by intraocular structures with different optical properties. The OCT uses coherent laser light to sweep the retina and analyze the light reflected by the retinal layers. This way, an in vivo "biopsy" of the retina provides information 
of high-quality resolution about all its layers. The OCT allows qualitative (localization, shape, structure) and quantitative analysis (retinal measurements, especially of the retinal thickness and retinal nerve fiber layer (RNFL) ${ }^{10,11}$.

RNFL thickness has been used to diagnose various diseases associated with ophthalmology and neurology ${ }^{12}$. Its use in the diagnosis or early prediction of anterior visual pathway lesions is also documented in various studies in the west during the last few years. A recently published Swiss study concluded that RNFL has a clear role in the diseases of visual pathway. They become more accurate when combined with the papillomacular bundle ${ }^{13}$. A study conducted on the patients of Non-Arteritic Anterior Ischemic Optic Neuropathy revealed that OCT has a significant role in determining the RNFL thickness and changes in RNFL thickness can be helpful in diagnosing and formulating the treatment plan for the underlying disease ${ }^{14}$. Rolle et al, conducted a study exclusively in patients of glaucoma and concluded that retinal cell layer showed significant changes and found RNFL reduced in these patients as compared to normal population ${ }^{15}$. A case series from Australia revealed that the integration of adjunct imaging and retinal nerve fiber layer thinning will assist clinicians to guide clinical investigations toward a likely diagnosis in the light of significant individual variations ${ }^{16}$.

Facility of OCT is available at selected centers of Pakistan. Moreover, usually there is less or no liaison between neurology and ophthalmology teams, so most neurologists are unaware of the utility of this non-invasive investigation in the local setup. Neuro-ophthalmological lesions put a significant burden on Pakistani health care system ${ }^{17}$. They also add a lot to the number of disability days faced by the patient especially diseases like tumors and glaucoma ${ }^{18}$. If OCT can be used to detect these lesions early or help in prevention or early diagnosis, it would be a revolution in the lives of patients. Therefore, the present study was planned with the objective to assess the role of quantification of retinal nerve fiber layer thickness for early detection of anterior visual pathway lesions and relationship of thinning of this layer with the presence of these lesions.

\section{METHODOLOGY}

A case-control study was designed and conducted at the Armed Forces Institute of Ophthalmology (AFIO), from January to July 2019. Keeping level of significance a at 5\%, power of the test $1-\beta$ at $80 \%$, anticipated probability of exposure given disease $\mathrm{P}^{*}$ at $0.485,4$ anticipated probability of exposure given no disease $p 2^{*}$ at 0.3 , a sample size of 99 was calculated, which was rounded off to 100 for each group. Non-probability consecutive sampling technique was used to collect the cases of anterior visual pathway lesions who were diagnosed clinically by the consultant ophthalmologist and neurologist at AFIO. After enrolling 100 cases, age and gender matched controls were selected from the community hospital attendants using nonprobability consecutive sampling technique. Healthy attendants of the patients admitted at AFIO were chosen as the controls for this study. Patients with lesions extending to posterior pathway or comorbid ocular or neurological problems or with malignant lesions were not included in the study. Controls with uncontrolled physical illnesses or having past history of ophthalmological or neurological illnesses were also excluded.

After ethical approval from the institutional review board and written informed consent from the potential participants including cases and controls, patients of anterior visual pathway lesions diagnosed at AFIO and matched controls fulfilling the above-mentioned selection criteria were included in the study. Data was collected on a structured proforma specially designed for this study. Detailed ophthalmological examination including slit lamp examination was performed on all the study participants including the controls as per protocol of the study. For all cases and controls, RNFL thickness was measured using OCT by a trained professional and measurements were added in the proforma for each individual.

Data was analyzed using SPSS version 23. Categorical variables such as gender and types of 
anterior visual pathway lesions were presented as frequency and percentages. Independent sample t-test was used to compare mean RNFL thickness between cases and controls. Binary logistic regression was used to calculate the odds of having anterior visual pathway lesion based on RNFL thickness, $p$-value $\leq 0.05$ was taken as significant.

\section{RESULTS}

A total of 100 cases and 100 controls were included in the study. Out of these, 109 (54.5\%) were male and 91 (45.5\%) were female. Mean age of the patients were $39.14 \pm 3.925$ years while mean age of the controls were $39.23 \pm 2.415$ years. The cases included 38 (38\%) glaucoma patients, 7 (7\%) pituitary tumors, $5(5 \%)$ compressive optic neuropathies, 4 (4\%) optic neuritis, 8 (8\%) NonArteritic Anterior Ischemic Optic Neuropathy, 8 (8\%) Anterior Ischemic Optic Neuropathy, 9 (9\%) toxic optic neuropathies, $8(8 \%)$ traumatic optic neuropathies, 4 (4\%) benign intracranial hypertension, $5(5 \%)$ hereditary optic neuropathy and 4 (4\%) disc drusens cases. Mean RNFL thickness among the case group was $72.21 \pm 9.62 \mu \mathrm{m}$ while among the control group was $101.34 \pm 9.61 \mu \mathrm{m}$. A significant difference was observed between cases and controls in terms of mean RNFL thickness $(p<0.001)$ as shown in table-I.

Table-I: Comparison of mean RFNL thickness between cases and controls.

\begin{tabular}{l|c|c|c}
\hline Parameter & $\begin{array}{c}\text { Cases } \\
(\mathbf{n}=\mathbf{1 0 0})\end{array}$ & $\begin{array}{c}\text { Controls } \\
(\mathbf{n}=\mathbf{1 0 0})\end{array}$ & $\begin{array}{c}p \text { - } \\
\text { value }\end{array}$ \\
\hline $\begin{array}{l}\text { Mean RFNL } \\
(\mu \mathrm{m})\end{array}$ & $72.2 \pm 9.62$ & $101.34 \pm 9.61$ & $<0.001$ \\
\hline
\end{tabular}

Table-II: Predictor variables for anterior visual pathway lesions.

\begin{tabular}{|c|c|c|}
\hline Predictor variables & $p$-value & $\begin{array}{c}\text { Odds Ratio } \\
\text { (95\% CI) }\end{array}$ \\
\hline Age (ref. <35 years) & 0.361 & $\begin{array}{c}0.713 \\
(0.345-1.472)\end{array}$ \\
\hline Gender (ref. male) & 0.402 & $\begin{array}{c}0.757 \\
(0.394-1.453)\end{array}$ \\
\hline RNFL (ref. $<85 \mu \mathrm{m}$ ) & $<0.001$ & $\begin{array}{c}15.915 \\
(6.278-40.346)\end{array}$ \\
\hline
\end{tabular}

A logistic regression was performed to ascertain the effect of RNFL thickness on the likelihood that participants have anterior visual pathway lesions (table-II). While RNFL thickness significantly correlated with presence of anterior visual pathway lesions $(p<0.001)$, age $(p=0.361)$ and gender $(p=0.402)$ did not yield any significant effect. Subjects with RNFL thickness $<85 \mu \mathrm{m}$ were 15.9 times more likely to exhibit anterior visual pathway lesions.

\section{DISCUSSION}

Anterior visual pathway lesions can present in number of ways and can exercise a huge effect on quality of life of the patients suffering from these lesions ${ }^{18}$. Treatment of anterior visual pathway lesions involves a multi-disciplinary approach involving an ophthalmologist and neurologist. This liaison usually lacks in the local population, causing a delay in diagnosis of such patients. Epidemiology of these lesions is uncertain in the local population, but few studies accomplished by neurologists and ophthalmologist in this regard revealed that these are not uncommon clinical conditions ${ }^{17}$. Early diagnosis and treatment have been the key in the management, so researchers have continuously tried to find a method for this purpose. Measuring RNFL thickness with the help of OCT is one such method which can help in early detection of these lesions?.

In the present study, mean RNFL thickness in controls was $101.34 \pm 9.61 \mu \mathrm{m}$. These findings were from any eye of the population with no neurological or ophthalmological abnormalities. On the other hand, mean RNFL thickness in eye of affected side of patients with anterior visual pathway lesions was $72.21 \pm 9.62 \mu \mathrm{m}$. The difference between the two was significant with $p$ $<0.001$. These results clearly depict that considerable thinning of retinal nerve fiber layer occurs among the patients with visual pathway lesions. Significant thinning of the retinal layer among the patients with different types of lesions of the visual pathway has been reported by other studies as well. Han et al, reported a significantly higher RNFL thickness in controls (114.82 \pm 10.96 $\mu \mathrm{m})$ as compared to cases $(64.73 \pm 10.65 \mu \mathrm{m})^{14}$. Similar results have also been reported by Rolle 
et al, who reported decreased RNFL thickness in glaucoma patients $(74.69 \pm 11.34 \mu \mathrm{m})$ than in healthier adults $(103.08 \pm 8.02 \mu \mathrm{m})^{15}$.

Glaucoma was the commonest diagnosis 38 (38\%) for which OCT was performed in the present study. Various studies in the past have discussed the fact that RNFL thickness has been found reduced in the patients of glaucoma ${ }^{15}$. Question arises that people who have thin RNFL at base line i.e, prior to the diagnosis of glaucoma or any lesion are at an increased risk of developing the disease or not?. Decreased RNFL thickness in patients was associated with an increased likelihood of presenting with these lesions. Toxic optic neuropathy was the second most common condition which was encountered in this study. Past studies have also revealed reduced thickness of RNFL among the patients of toxic optic neuropathy which was supported by the present analysis as well ${ }^{8}$.

Increasing age or gender was not a consistent correlation with the presence of anterior visual pathway lesions in the present study. Matched cases and controls on parameters of age and gender were included. No correlation was found of these parameters with presence of lesion. These results were in accordance with those of Rolle et al, who reported no significant effect of age or gender on study parameters $(p=0.24 ; 0.16)^{15}$. It could also be concluded that it was only thickness of RNFL which affected the presence or absence of anterior visual pathway lesion in the present analysis and no other factor predicted this association.

OCT emerged is a reliable technique in order to early detect and manage the anterior visual pathway lesions. Currently, it is used as a supportive investigation after clinical diagnosis of any lesion 7,16 , but if other studies also support the findings of this study, then this noninvasive investigation modality can be incorporated in diagnosis or screening of neurological as well as ophthalmological lesions. RNFL thickness can be a reliable screening parameter and can be a good tool for primary prevention of anterior visual pathway lesions.

It remains unclear that decrease in retinal nerve fiber layer is the cause of anterior visual pathway lesions or has been the result of these lesions. Moreover, mean RNFL thickness of four quadrants was taken as a parameter in this study. It would have been better if length of all four quadrants was measured separately and compared. In future, a community-based cohort study may be conducted by following up the subjects with decreased RNFL thickness till they develop any lesion to make this association clearer.

\section{CONCLUSION}

Decreased retinal nerve fiber layer thickness can serve as a predictor for the anterior visual pathway lesions. Optical coherence tomography should be incorporated for routine screening of high-risk cases in order to identify anterior visual pathway lesions in time.

\section{CONFLICT OF INTEREST}

This study has no conflict of interest to be declared by any author.

\section{REFERENCES}

1. Grover AK, Honavar SG, Azad R, Verma L. A national curriculum for ophthalmology residency training. Ind J Ophthalmol 2018; 66(6): 752-83.

2. Heckmann JG, Vachalova I, Lang CJG, Pitz S. Neuro-Ophthalmology at the Bedside: A Clinical Guide. J Neurosci Rural Pract 2018; 9(4): 561-73.

3. Donohue MW, Cohen JH, Cronin TW. Cerebral photoreception in mantis shrimp. Sci Rep 2018; 8(1): 9689-98.

4. Frau J, Fenu G, Signori A, Coghe G, Lorefice L, Barracciu MA, et al. A cross-sectional and longitudinal study evaluating brain volumes, RNFL, and cognitive functions in MS patients and healthy controls. BMC Neurol. 2018; 18(1):67-74.

5. Pillay G, Ganger A, Singh D, Bhatia R, Sharma P, Menon V, et al. Retinal nerve fiber layer and ganglion cell layer changes on optical coherence tomography in early multiple sclerosis and optic neuritis cases. Ind J Ophthalmol 2018; 66(1): 114-19.

6. Van Manen L, Dijkstra J, Boccara C, Benoit E, Vahrmeijer AL, Gora MJ, et al. The clinical usefulness of optical coherence tomography during cancer interventions. J Cancer Res Clin Oncol 2018; 144(10): 1967-90.

7. Wylęgała A. Principles of OCTA and Applications in Clinical Neurology. Curr Neurol Neurosci Rep 2018; 18(12): 96-06.

8. Iorga RE, Moraru A, Ozturk MR, Costin D. The role of Optical Coherence Tomography in optic neuropathies. Rom J Ophthalmol 2018; 62(1): 3-14.

9. Wang L, Murphy O, Caldito NG, Calabresi PA, Saidha S. Emerging applications of optical coherence tomography angiography 
(OCTA) in neurological research. Eye Vis (Lond) 2018; 5(1): 11-21.

10. Cheong KX, Lim LW, Li KZ, Tan CS. A novel and faster method of manual grading to measure choroidal thickness using optical coherence tomography.Eye (Lond) 2017; 32(2): 433-38.

11. Philip AM, Gerendas BS, Zhang L, Faatz H, Podkowinski D, Bogunovic $\mathrm{H}$, et al. Choroidal thickness maps from spectral domain and swept source optical coherence tomography: algorithmic versus ground truth annotation. Br J Ophthalmol 2016; 100(10): 1372-76.

12. Costello F. Retinal imaging with optical coherence tomography: a biomarker in multiple sclerosis?.Eye Brain 2018; 10(1): 47-63.

13. Zehnder S, Wildberger H, Hanson JVM, Lukas S, Pelz S, Landau $\mathrm{K}$, et al. Retinal Ganglion Cell Topography in Patients With Visual Pathway Pathology. J Neuroophthalmol 2018; 38(2): 172-78.

14. Han M, Zhao C, Han QH, Xie S, Li Y. Change of retinal nerve layer thickness in non-arteritic anterior ischemic optic neuropathy revealed by fourier domain optical coherence tomography. Curr Eye Res 2016; 41(8): 1076-81.

15. Rolle T, Dallorto L, Tavassoli M, Nuzzi R. Diagnostic ability and discriminant values of oct-angiography parameters in early glaucoma diagnosis. Ophthalmic Res 2018; 28(1): 1-10.

16. Zangerl B, Whatham A, Kim J, Choi A, Assaad NN, Hennessy $\mathrm{MP}$, et al. Reconciling visual field defects and retinal nerve fibre layer asymmetric patterns in retrograde degeneration: an extended case series. Clin Exp Optom 2016; 100(3): 214-26.

17. Alswailmi FK. Global prevalence and causes of visual impairment with special reference to the general population of Saudi Arabia. Pak J Med Sci 2018; 34(3): 751-56.

18. Kumar S, Ichhpujani P, Singh R, Thakur S, Sharma M, Nagpal N. The impact of primary open-angle glaucoma: Quality of life in Indian patients. Ind J Ophthalmol 2018; 66(3): 416-19. 